\title{
Neurosurgical training and global health education: systematic review of challenges and benefits of in-country programs in the care of neural tube defects
}

\author{
Kellen Gandy, PhD, ${ }^{1}$ Heidi Castillo, MD, ${ }^{2}$ Brandon G. Rocque, MD, MS, ${ }^{3}$ Viachaslau Bradko, MD, ${ }^{4}$ \\ William Whitehead, MD, MPH, ${ }^{5}$ and Jonathan Castillo, MD, MPH ${ }^{2}$ \\ ${ }^{1}$ Hematology-Oncology and 2Developmental Pediatrics, Department of Pediatrics, Baylor College of Medicine, Houston, Texas; \\ ${ }^{3}$ Department of Neurosurgery, University of Alabama at Birmingham, Alabama; and Departments of ${ }^{4}$ Orthopedic Surgery and \\ ${ }^{5}$ Pediatric Neurosurgery, Baylor College of Medicine, Houston, Texas
}

\begin{abstract}
OBJECTIVE The recognition that neurosurgeons harbor great potential to advocate for the care of individuals with neural tube defects (NTDs) globally has sounded as a clear call to action; however, neurosurgical care and training in lowand middle-income countries (LMICs) present unique challenges that must be considered. The objective of this study was to systematically review publications that describe the challenges and benefits of participating in neurosurgeryrelated training programs in LMICs in the service of individuals with NTDs.

METHODS Using MEDLINE (PubMed), the authors conducted a systematic review of English- and Spanish-language articles published from 1974 to 2019 that describe the experiences of in-country neurosurgery-related training programs in LMICs. The inclusion criteria were as follows-1) population/exposure: US residents, US neurosurgeons, and local incountry medical staff participating in neurosurgical training programs aimed at improving healthcare for individuals with NTDs; 2) comparison: qualitative studies; and 3) outcome: description of the challenges and benefits of neurosurgical training programs. Articles meeting these criteria were assessed within a global health education conceptual framework. RESULTS Nine articles met the inclusion criteria, with the majority of the in-country neurosurgical training programs being seen in subregions of Africa (8/9 [89\%]) and one in South/Central America. US-based residents and neurosurgeons who participated in global health neurosurgical training had increased exposure to rare diseases not common in the US, were given the opportunity to work with a collaborative team to educate local healthcare professionals, and had increased exposure to neurosurgical procedures involved in treating NTDs. US neurosurgeons agreed that participating in international training improved their own clinical practices but also recognized that identifying international partners, travel expenses, and interference with their current practice are major barriers to participating in global health education. In contrast, the local medical personnel learned surgical techniques from visiting neurosurgeons, had increased exposure to intraoperative decision-making, and were given guidance to improve postoperative care. The most significant challenges identified were difficulties in local long-term retention of trained fellows and staff, deficient infrastructure, and lower compensation offered for pediatric neurosurgery in comparison to adult care.
\end{abstract}

CONCLUSIONS The challenges and benefits of international neurosurgical training programs need to be considered to effectively promote the development of neurosurgical care for individuals with NTDs in LMICs. In this global health paradigm, future work needs to investigate further the in-country professionals' perspective, as well as the related outcomes. https://thejns.org/doi/abs/10.3171/2019.12.FOCUS19448

KEYWORDS neural tube defect; spina bifida; myelomeningocele; global health; guidelines; international health

$\mathrm{N}$ EURAL tube defects (NTDs) are associated with a significant burden of death and disability worldwide. In the US, NTDs account for nearly 6 of 10,000 live births, ${ }^{26}$ a rate that has been attained in part through the implementation of guidelines for periconceptional folic acid intake. ${ }^{7}$ However, the prevalence of NTDs is much higher in low- and middle- income countries (LMICs), where recommendations for folic acid intake (i.e., $400 \mu \mathrm{g}$ per day) to reduce the risk of NTDs are poorly adhered to, and divergent prevalence rates exist within many nations. ${ }^{5}$ Per 10,000 births, the prevalence rate of NTDs in Africa ranges from 5.2 to 75.4 with a median of 11.7; in

ABBREVIATIONS LMIC = low- and middle-income country; NTD = neural tube defect; $\mathrm{QOL}=$ quality of life.

SUBMITTED May 31, 2019. ACCEPTED December 20, 2019.

INCLUDE WHEN CITING DOI: 10.3171/2019.12.FOCUS19448. 
Southeast Asia, from 1.9 to 66.2 with a median of 15.8 ; and in South/Central America, from 11.9 to 27.9 with a median of 19.9. ${ }^{28}$ Concurrently on the world stage, an integral part of the approach to NTDs is assertive, proactive, informed public health advocacy by neurosurgeons. The recognition that, along with care and education, neurosurgeons harbor great potential to advocate for science-based primary prevention - through fortification-has sounded as a clear call to action. ${ }^{11}$

Pediatric neurosurgeons in North America acknowledge the need for improved education in treating NTDrelated conditions such as occult spinal dysraphism and tethered cord, as well as other neurosurgical conditions, including craniosynostosis and hydrocephalus. ${ }^{3}$ While improved education is needed in the US, this need is even greater among LMICs, where these conditions are more prevalent. ${ }^{16}$ Concomitantly, to improve global health education, the Accreditation Council for Graduate Medical Education has supported the incorporation of global health training among various subspecialty residency programs. In fact, the majority of internal medicine residency programs now offer global health training in their curriculum. ${ }^{15}$ While global health training is less commonly offered in surgical programs ${ }^{18}$ US residents in general surgery programs have demonstrated a strong interest in participating in international training ${ }^{21}$ and have reported that they benefit from international rotations when given the opportunity to serve in limited-resource settings. ${ }^{13}$ Global health educational opportunities are most commonly offered in emergency medicine, general surgery, and preventive medicine training and are less commonly available in orthopedic surgery, psychiatry, and obstetrics and gynecology residency training programs. ${ }^{12}$ Moreover, US resident interest in global health education and international rotations has grown exponentially over the last decade..$^{10}$ With increasing cultural diversity in the US, it is essential that residents better understand the etiology and global burden of diseases, recognize the disparities among global health care systems, and consider the influence of culturally diverse social determinants of health unique to LMICs. To improve global health care education available to trainees, it is important to consider the socioeconomic challenges and educational benefits of participating in incountry global health training. The term "in-country" is in reference to LMIC medical sites that provide medical services to the local population.

Given the escalating interest in global health training and the high prevalence of NTDs in LMICs, it is important that in-country neurosurgical training programs continue to develop and remain current with evolving surgical techniques to serve this population. However, socioeconomic and geographical factors have impacted the uneven international development of neurosurgical care for individuals with NTDs, with higher-income countries having more established treatment protocols, greater resources, and lower incidences of NTDs. ${ }^{28}$ The employment of neurosurgery education in under-resourced settings presents unique challenges, and these challenges must be considered when developing and participating in neurosurgical training programs in these regions. The objective of this study was to systematically review publications that de- scribe the challenges and benefits of in-country neurosurgical training programs from the perspective of visiting US residents, US neurosurgeons, and local medical professionals.

\section{Methods \\ Search Criteria and Strategy}

A systematic review was conducted in accordance with the PRISMA guidelines, and registered in the PROSPERO registry (CRD 42019130176; April 29, 2019). ${ }^{19}$ Using the MEDLINE (PubMed) database, we performed a search of all published articles (from 1974 to 2019) using the following terms: "neural tube defect" or "spinal dysraphism" or "spina bifida" or "myelomeningocele" AND "global health" OR "mission*" OR "international health" OR "global surgery" OR "global neurosurgery" OR "internship" OR "residency" OR "resident" OR "education" OR "educator" OR "curriculum" OR "teaching" OR "elective" OR "rotation." The results were restricted to English- and Spanish-language publications. The search was completed using the MEDLINE (PubMed) database on April 3, 2019.

The inclusion criteria were as follows-1) population/ exposures: US residents, US neurosurgeons, and healthcare staff in LMICs participating in neurosurgical training programs to improve healthcare for individuals with NTDs; 2) comparison: qualitative studies; and 3) outcome: description of the challenges and benefits of in-country neurosurgical training programs. Review articles and conference abstracts were excluded. An initial screening of titles and abstracts was conducted by two independent reviewers to determine which articles met the inclusion criteria. A subsequent secondary screening of full-text articles was conducted, and any discrepancies related to article eligibility were presented to the coauthors and a consensus was reached through discussion.

\section{Results}

Four hundred seventy-eight articles were identified in accordance with PRISMA guidelines, of which 9 articles met the inclusion criteria (Fig. 1). The majority of the incountry neurosurgical training programs were found in subregions of Africa (8/9 [89\%]), with 1 (11\%) in South/ Central America. $1,2,8,9,16,17,20,22,27$

\section{US Residents' and Neurosurgeons' Perspectives}

US-based residents and neurosurgeons who participated in global health neurosurgical training had increased exposure to patients with NTDs not as commonly encountered in the US, were given the opportunity to work with a collaborative team and educate the local medical staff, and had increased exposure to neurosurgical procedures involved in treating NTDs. ${ }^{17,22}$ Barriers to participating in global neurosurgical training programs to treat NTDs were not explicitly mentioned for US residents. In addition, US neurosurgeons acknowledged that participating in global health training improved their clinical practices and gave them increased exposure to rare pathology; they also recognized that identifying international part- 


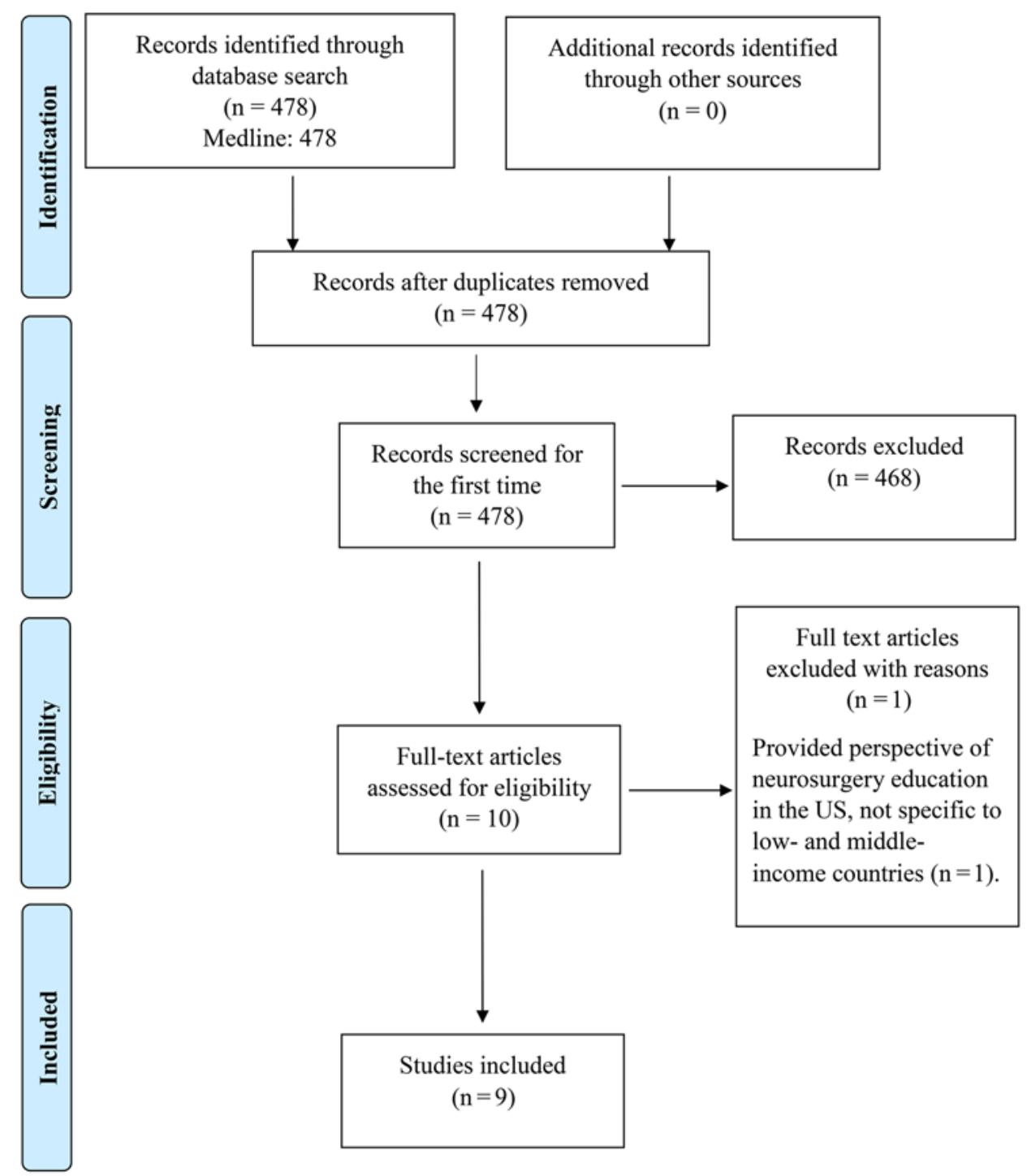

FIG. 1. Flow diagram illustrating our search strategy in accordance with PRISMA guidelines.

ners, travel expenses, and interference with current practice were major barriers to participating in global health education. ${ }^{8}$ US neurosurgeons acknowledged that a lack of resources in these regions (i.e., deficient infrastructure, lack of imaging equipment, citizens' low incomes, and high cost associated with treatment) and cultural factors can limit treatment options. ${ }^{1,2}$ Moreover, it has been recognized that the surgeon's perception of the patient's quality of life (QOL) contributes to the decision-making process as to whether or not to surgically intervene. ${ }^{2}$ Overall, the majority of US-based residents and neurosurgeons participated in 2- to 6-week rotations.

\section{Local Medical Personnel Perspectives}

None of the articles included in this systematic review were written directly from the perspective of the local medical personnel, patients, or healthcare system. Instead, the perspective of the local medical personnel was pro- vided through proxy reports from visiting neurosurgical teams or collaborating institutions. . $2,9,16,17,20,27$ The residing in-country medical staff members were thought to learn surgical techniques from visiting neurosurgeons, including proper sterilization, had increased exposure to intraoperative decision-making, and were given guidance to improve postoperative care. ${ }^{17,20,27}$ Academic training sessions, which included access to internet resources, peer-reviewed papers, and online webinars, improved education and promoted greater confidence in treating patients. ${ }^{16}$ The most significant challenges identified were difficulties in local long-term retention of trained fellows and staff, deficient infrastructure, lack of guidelines, poor selfconfidence among local physicians, and lower compensation offered for pediatric neurosurgery in comparison to adult care. ${ }^{1,2,16,20}$ The in-country neurosurgical training programs ranged in duration from 8 weeks to 1 year.

Although in practice the identified articles reflect only a few dedicated neurosurgical training programs, a sum- 
TABLE 1. Perspective of US residents, US neurosurgeons, and in-country medical professionals on global health neurosurgery training specifically relevant to NTD education

Authors \& Year Educational Context \& Location

Mainthia et al., 2009 US pediatric neurosurgeon mission trips to South \& Central America (12 years of experience). Host/coordinating program(s): The Pediatric Foundation of Guatemala, Rotary Club of St. Vincent \& the Grenadines. Host hospital: The Hospital Nacional Hermano Pedro de Bethancourt in Guatemala. Coordinating organization btwn Virginia Commonwealth University \& the World Pediatric Project.

Wilson et al., $2012 \quad$ Local resident physicians \& neurosurgeons at a training program (15 weeks) in Tanzania, Africa. Host/coordinating program(s): Barrow Neurological Institute. Host hospital: Bugando Medical Centre.

Albright \& Ferson, 2012; Missionary pediatric neurosurgery in Kenya, Africa.

Albright, $2016 \quad$ Host/coordinating program(s): Medtronic Foundation \& the International Society of Pediatric Neurosurgery. Host hospital: Kijabe Hospital.

Rocque \& Lam, 2014 US resident experiences w/ international neurosurgery in Kenya, Africa (2- to 4-week rotations). Host/coordinating program(s): Medtronic Foundation \& the International Society of Pediatric Neurosurgery. Host hospital: Kijabe Hospital.

Piquer et al., $2015 \quad$ Local volunteer neurosurgeons evaluate results of surgical training \& medical care in East, Central, \& South Africa (7 years). Host/coordinating program(s): Neurosurgery Education and Development (NED) Foundation, the Foundation for International Education in Neurological Surgery (FIENS).

Host hospitals: Kenyatta Hospital, Nyeri Provincial Hospital, Aga Khan Hospital, Aga Khan University Hospital, Coast Provincial General Hospital, Kijabe Mission Hospital, Muhimbili Orthopaedic Institute, Bethel Teaching General Hospital, Black Lion Hospital, Kigali National Hospital, Mnanzi Mmoja Hospital, Moi Teaching and Referral Hospital, Mulango Hospital Complex, Myunsung Christian Mission, Getrude's Garden Children's Hospital, Mewa Hospital, Neurspine Center, Police Hospital, Bugando Teaching Hospital, Harare Central Hospital.

Davis et al., $2017 \quad$ US-based neurosurgeons completed surveys about global neurosurgical outreach in Africa \& South America. Host/coordinating program(s): CURE Uganda, World Pediatric Project, Foundation for International Education in Neurological Surgery (FIENS), Project Medishare, ISPN, World Medical Mission, BethanyKids, AMPATH Consortium Kenya, WFNS, World Craniofacial Foundation, Solidarity Bridge, SMILES, Shiners, Samaritan's Purse, Project Perfect World, Neurosurgery Outreach Foundation, Maghreb-American Health Foundation, INCA, Indian Society of Pediatric Neurosurgery, Healing the Children, Haiti Healthy Kids, Global Neuro Rescue, Children's of Alabama Global Health, Children's China Foundation, ASPN, AANS/CNS Joint Section on Pediatric Section.

Host hospital: not provided.

Dewan et al., $2018 \quad$ Local fellows' experience at a neurosurgery training program (8 weeks) in Uganda, Africa. Host/coordinating program(s): CURE Hydrocephalus and Spina Bifida (CHSB) fellowship. Host hospital: CURE Children's Hospital of Uganda.

Leidinger et al., 2018 Volunteer neurosurgeon coordinator in Zanzibar, Africa (18 weeks).

Host/coordinating program(s): Neurosurgery Education and Development (NED) Foundation, the Foundation for International Education in Neurological Surgery (FIENS), the College of Surgeons of East, Central, \& Southern Africa (COSECSA). Host hospital: Mnazi Mmoja Hospital.

For the complete version of this table, see the Supplemental Table.

mary of the benefits and challenges described by various medical providers in these programs is presented in Table 1.

\section{Discussion}

Further development of global health neurosurgical training is necessary to reduce the worldwide burden of NTDs, particularly in LMICs. The purpose of this study was to review the benefits and challenges of neurosurgical education in LMICs, with a specific focus on treatment of NTDs. While there is growing interest in the US to promote global health education, residents are met with barriers that limit their involvement in international training, such as lack of funding, limited elective time, and the requirement to provide clinical services at their local insti- tution. ${ }^{15}$ Neurosurgeons in the US also acknowledge that interference with current practice, travel expenses, and difficulty in establishing reliable international collaborations are major barriers to participating in global health. ${ }^{1,8}$ However, these challenges are also accompanied by many benefits for US residents and neurosurgeons participating in international rotations or medical missions. ${ }^{8,22}$ Mainly, US residents and neurosurgeons gain valuable insight into how socioeconomic factors specific to LMICs influence decision-making for neurosurgical treatment and followup care for NTDs. ${ }^{1,2}$ They also benefit from the opportunity to work with a collaborative team to educate local professionals and caregivers. ${ }^{17}$ Additionally, they have increased exposure to rare diseases and the surgical techniques needed to treat these disorders. ${ }^{1,2}$ In fact, the average exposure of US resident trainees to pediatric spinal 
dysraphism is approximately 8.1 cases (SD 11) for the entire neurosurgical residency training program. In contrast, US residents participating in international pediatric neurosurgery rotations at a pediatric hospital in Kenya were exposed to 4 cases of spinal dysraphism per week, with the majority presenting as myelomeningocele. ${ }^{22}$

From the perspective of the visiting neurosurgeons, the local medical staff members were thought to have gained several benefits from participating in the neurosurgeryrelated training programs. In particular, the local medical staff benefited from learning surgical techniques and were given guidance to improve postoperative care for individuals requiring neurosurgery. These improvements and recommendations for care allowed residing medical professionals to subsequently conduct successful surgeries on local individuals, even in the absence of the visiting neurosurgical team. ${ }^{17}$ The local medical staff were also trained on proper sterilization and equipment maintenance. The presence of a neurosurgical training program inspired other health project developments in the local regions ${ }^{22}$ and improved confidence in the treatment for the residing medical staff. ${ }^{16}$ In contrast, the most significant challenges identified for residing medical staff were difficulties in long-term retention of locally training fellows and staff, deficient infrastructure, lack of an ability to repair equipment, and lower compensation offered for pediatric neurosurgery in comparison to adult care..$^{2,9,16,27}$ Last, while 1-year fellowships were made available at some sites, only a few applicants were able to successfully complete the program, suggesting that long-term training programs may be less effective in the initial development of neurosurgical training platforms. ${ }^{1,2}$

Given the high prevalence of NTDs in many LMICs, the available relevant literature has been systematically reviewed with the hope of understanding current efforts in international neurosurgical education. While all of the identified programs incorporate broad general neurosurgical training, we specifically described the experiences of treating NTDs in this context. Although in-country training programs have resulted in better treatment outcomes for residing individuals, ${ }_{17}^{17}$ special consideration must be given to the cultural and socioeconomic factors that impact neurosurgical care. Cultural influences, such as the belief that delivering unfavorable news is taboo, as observed in Kenya, can lead local medical staff to avoid telling families about potential illnesses or diseases, leaving many children to go untreated. ${ }^{2}$ Additionally, in rural areas of Africa, individuals seem to have a strong community-oriented mindset that can lead families to reject or hide members with disabilities, who contribute less to communal tasks, thus delaying or avoiding treatment. ${ }^{16}$ Socioeconomic factors influence neurosurgical treatment recommendations and guidelines. For instance, it is commonplace in the US to use preoperative imaging (i.e., MRI, etc.) to justify the need for surgical intervention in NTDs; however, the same resources are not available in LMICs, ${ }^{1,2}$ and alternative guidance for making a decision to operate need to be considered. The use of a mobile neuroendoscopic training intervention may be an appropriate alternative, given its established feasibility to improve patient outcomes and training opportunities for medical staff in rural areas of Africa. ${ }^{20}$ In addition, the significant costs associated with neurosurgical treatment in low-resource countries often leads families to first seek care from traditional medicine practitioners who utilize non-evidence-based practices, which can delay surgical treatment. ${ }^{2}$

The costs of treatment can also financially jeopardize a family or an entire village depending on the procedure. For this reason, the cost of treatment is often weighed against an individual's perceived QOL posttreatment. In fact, the treating surgeon and/or medical personnel will often consider a child's perceived degree of subsequent "meaningful life" (i.e., QOL) to initially determine whether or not to treat the child, as described by Leland Albright, a US neurosurgeon who has dedicated his career to improving pediatric neurosurgery in LMICs. ${ }^{2}$ Therefore, it is important that some awareness of the appropriate QOL measures be incorporated into neurosurgical training programs and the subsequently needed community-based rehabilitation. ${ }^{14}$

\section{Educational Lessons for Global Neurosurgical Training}

1. US residents and neurosurgeons should expect to encounter a large number of NTD cases during international rotations to low- and middle-income geographical regions. Therefore, US residency program directors should advocate for specific education on how to care for individuals with NTDs and how socioeconomic factors can influence treatment options.

Global neurosurgical programs to treat NTDs are sparsely reported in the literature and often limited by deficient infrastructure, limited resources, and lack of consistent in-country medical staff. However, these programs benefit from global collaborations, which seem to improve the skillset of local medical professionals in LMICs. Although at this time the relevant literature is sparse, it could be hoped that in time there will be the needed documentation of improved outcomes resulting from these partnerships. To establish global collaborations that benefit in-country training, physicians interested in global health outreach can begin by contacting organizations such as the World Pediatric Project (www.worldpediatricproject.org); the Neurosurgery, Education, and Development Foundation (www.nedfoundation.wordpress.com); Samaritan's Purse International Relief (www.samaritanspurse.org); or the Foundation for International Education in Neurological Surgery (www.fiens.org) to engage in active collaborations or to establish new partnerships. Additionally, to participate in ongoing educational endeavors a number of nongovernmental organizations with special focus on neurosurgical care, such as the International Federation for Spina Bifida and Hydrocephalus (www.ifglobal.org) and CURE Neuro (www.cure.org/neuro), can be contacted to identify specific areas of service and projects in LMICs.

2. Medical providers involved in global neurosurgery should describe and publish their experiences to foster the development of evidence-based guidelines for treating NTDs in LMICs. Perspectives of the local health care systems and reports of patient outcomes prior to and following the implementation of neurosurgical training programs have yet to be reported in the lit- 
erature, demonstrating a great need for local medical personnel to disseminate their experiences. Each region presents with unique benefits and challenges that should be systematically reviewed and incorporated into global health education. In the new global health paradigm, neurosurgeons have a role in educating others internationally regarding the recently updated "Guidelines for the Care of People with Spina Bifida" (available at: https://www.spinabifidaassociation.org/ resource/guidelines/). Neurosurgeons can advocate for guideline-based management and can leverage these resources to inform and guide care in a number of key issues (e.g., fortification, prenatal repair, tethered cord syndrome, QOL, neurodevelopment/neuropsychology, skin injury, and transitions across the continuum of care, etc.) where applicable abroad. ${ }^{4}$

3. Additionally, the role for neurosurgeons in providing education regarding QOL is critical, for among individuals with NTDs, QOL is an underrepresented area of surgical education. Both neurosurgeons and medical providers in community-based rehabilitation centers should use standardized QOL measures appropriate for NTD populations to improve overall health outcomes. Metrics of QOL can be used preoperatively to make informed decisions regarding the benefit of neurosurgical treatment. Standardized assessments such as the KIDSCREEN-10 (www.kidscreen.com) and QUALAS ${ }^{23-25}$ may be suitable metrics for measuring QOL in individuals with NTDs in these settings. Finally, linguistically congruous (local language) study of QOL has enlarging relevance amid not only immigrants and refugees in higher-income countries, but also clearly among internally displaced persons in LMICs. ${ }^{6}$

\section{Limitations}

We acknowledge several limitations common to systematic reviews and the nature of synthesizing large data sets. To reduce these limitations, PRISMA guidelines were followed. A second limitation of this review is that articles were only reviewed if published in English or Spanish, which may help explain why no articles were found in Southeast Asia despite a high prevalence of NTDs. Then again, a substantial paucity of collaborative development of training programs in the region could not be ruled out. The heterogeneity of pathology within this population (i.e., NTDs, hydrocephalus, tethered cord syndrome, cranial trauma, etc.) also represents another limitation. However, only neurosurgical training programs that reported outcomes for NTDs were included. Relatively few publications met our inclusion criteria, making it difficult to make strong conclusions, but the qualitative nature of the studies provided needed initial insight into global health training for neurosurgery-related programs. Last, no publications were written directly from the perspective of the local medical personnel, but rather, proxy-reported perspectives were provided by the visiting neurosurgical team or collaborating institution in North America traveling to LMICs. It is critical for surgeons in LMICs to report on their experiences to provide a more holistic understanding of how international training efforts are influencing local medical practice. However, these findings may reflect a lack of available resources needed to publish an article in a peer-reviewed journal. To address these limitations, global health educators should encourage participants to describe and publish their unique experiences to improve education and healthcare.

\section{Conclusions}

The various perspectives of the challenges and benefits of in-country neurosurgical training programs must be considered to effectively promote the development of neurosurgical care for individuals with NTDs in LMICs. Future work needs to further investigate the in-country professionals' perspective, as well as the related outcomes. Special consideration for cultural and socioeconomic influences (i.e., social determinants of health, such as language, culture, and immigration) is warranted when USbased surgeons are involved in service and training in LMICs. Although neurosurgical care and training abroad present unique challenges, it is evident that neurosurgeons harbor the potential to advocate for and to serve this population through surgical education globally.

\section{Acknowledgments}

We are deeply grateful for the countless individuals and their family members who participated in the research reviewed, without whom progress in the field would not be possible.

\section{References}

1. Albright AL: Reflections on developing pediatric neurosurgery in Sub-Saharan Africa. J Neurosurg Pediatr 18:127138,2016

2. Albright AL, Ferson SS: Developing pediatric neurosurgery in a developing country. J Child Neurol 27:1559-1564, 2012

3. Aldana PR, Steinbok P: Prioritizing neurosurgical education for pediatricians: results of a survey of pediatric neurosurgeons. J Neurosurg Pediatr 4:309-316, 2009

4. Bradko V, Castillo H, Janardhan S, Dahl B, Gandy K, Castillo J: Towards guideline-based management of tethered cord syndrome in spina bifida: a global health paradigm shift in the era of prenatal surgery. Neurospine 16:715-727, 2019

5. Castillo J, Castillo H: Global health and chronic non-communicable conditions: spina bifida care across a worldwide community. J Pediatr Rehabil Med 10:167-168, 2017

6. Castillo J, Gandy K, Bradko V, Castillo H: Language and Latino immigrants living with spina bifida: social determinants of health - the missing dimension in quality of life research. J Pediatr Rehabil Med 14:347-359, 2019

7. Crider KS, Bailey LB, Berry RJ: Folic acid food fortification-its history, effect, concerns, and future directions. Nutrients 3:370-384, 2011

8. Davis MC, Rocque BG, Singhal A, Ridder T, Pattisapu JV, Johnston JM Jr: State of global pediatric neurosurgery outreach: survey by the International Education Subcommittee. J Neurosurg Pediatr 20:204-210, 2017

9. Dewan MC, Onen J, Bow H, Ssenyonga P, Howard C, Warf BC: Subspecialty pediatric neurosurgery training: a skillbased training model for neurosurgeons in low-resourced health systems. Neurosurg Focus 45(4):E2, 2018

10. Drain PK, Primack A, Hunt DD, Fawzi WW, Holmes KK, Gardner P: Global health in medical education: a call for more training and opportunities. Acad Med 82:226-230, 2007

11. Estevez-Ordonez D, Davis MC, Hopson B, Arynchyna A, 
Rocque BG, Fieggen G, et al: Reducing inequities in preventable neural tube defects: the critical and underutilized role of neurosurgical advocacy for folate fortification. Neurosurg Focus 45(4):E20, 2018

12. Hau DK, Smart LR, DiPace JI, Peck RN: Global health training among U.S. residency specialties: a systematic literature review. Med Educ Online 22:1270020, 2017

13. Henry JA, Groen RS, Price RR, Nwomeh BC, Kingham TP, Hardy MA, et al: The benefits of international rotations to resource-limited settings for U.S. surgery residents. Surgery 153:445-454, 2013

14. Jimenez-Gomez A, Castillo H, Burckart C, Castillo J: Endoscopic third ventriculostomy to address hydrocephalus in Africa: a call for education and community-based rehabilitation. J Pediatr Rehabil Med 10:267-273, 2017

15. Kolars JC, Halvorsen AJ, McDonald FS: Internal medicine residency directors perspectives on global health experiences. Am J Med 124:881-885, 2011

16. Leidinger A, Extremera P, Kim EE, Qureshi MM, Young PH, Piquer J: The challenges and opportunities of global neurosurgery in East Africa: the Neurosurgery Education and Development model. Neurosurg Focus 45(4):E8, 2018

17. Mainthia R, Tye GW, Shapiro J, Doppenberg EM, Ward JD: A model for neurosurgical humanitarian aid based on 12 years of medical trips to South and Central America. J Neurosurg Pediatr 4:4-9, 2009

18. Mitchell KB, Tarpley MJ, Tarpley JL, Casey KM: Elective global surgery rotations for residents: a call for cooperation and consortium. World J Surg 35:2617-2624, 2011

19. Moher D, Liberati A, Tetzlaff J, Altman DG, PRISMA Group: Preferred reporting items for systematic reviews and meta-analyses: the PRISMA statement. BMJ 339:b2535, 2009

20. Piquer J, Qureshi MM, Young PH, Dempsey RJ: Neurosurgery Education and Development program to treat hydrocephalus and to develop neurosurgery in Africa using mobile neuroendoscopic training. J Neurosurg Pediatr 15:552-559, 2015

21. Powell AC, Mueller C, Kingham P, Berman R, Pachter HL, Hopkins MA: International experience, electives, and volunteerism in surgical training: a survey of resident interest. J Am Coll Surg 205:162-168, 2007

22. Rocque BG, Lam S: A case for international neurosurgical experience: US resident experiences with pediatric spinal dysraphism cases. Neurol Res 36:903-905, 2014

23. Szymański KM, Misseri R, Whittam B, Casey JT, Yang DY, Raposo SM, et al: Validation of QUALAS-T, a health-related quality of life instrument for teenagers with spina bifida. Cent European J Urol 70:306-313, 2017

24. Szymanski KM, Misseri R, Whittam B, Raposo SM, King SJ, Kaefer M, et al: QUAlity of Life Assessment in Spina bifida for Adults (QUALAS-A): development and international validation of a novel health-related quality of life instrument. Qual Life Res 24:2355-2364, 2015
25. Szymanski KM, Misseri R, Whittam B, Yang DY, Raposo SM, King SJ, et al: Quality of Life Assessment in Spina Bifida for Children (QUALAS-C): development and validation of a novel health-related quality of life instrument. Urology 87:178-184, 2016

26. Williams J, Mai CT, Mulinare J, Isenburg J, Flood TJ, Ethen M, et al: Updated estimates of neural tube defects prevented by mandatory folic acid fortification-United States, 19952011. MMWR Morb Mortal Wkly Rep 64:1-5, 2015

27. Wilson DA, Garrett MP, Wait SD, Kucia EJ, Saguda E, Ngayomela I, et al: Expanding neurosurgical care in Northwest Tanzania: the early experience of an initiative to teach neurosurgery at Bugando Medical Centre. World Neurosurg 77:32-38, 2012

28. Zaganjor I, Sekkarie A, Tsang BL, Williams J, Razzaghi H, Mulinare J, et al: Describing the prevalence of neural tube defects worldwide: a systematic literature review. PLoS One 11:e0151586, 2016

\section{Disclosures}

The authors report no conflict of interest concerning the materials or methods used in this study or the findings specified in this paper.

\section{Author Contributions}

Conception and design: J Castillo, Gandy, H Castillo, Rocque. Acquisition of data: J Castillo, Gandy, H Castillo, Bradko. Analysis and interpretation of data: J Castillo, Gandy, H Castillo, Rocque, Bradko. Drafting the article: J Castillo, Gandy, H Castillo. Critically revising the article: all authors. Reviewed submitted version of manuscript: all authors. Approved the final version of the manuscript on behalf of all authors: J Castillo. Study supervision: J Castillo.

\section{Supplemental Information}

\section{Online-Only Content}

Supplemental material is available online. Supplemental Table. https://thejns.org/doi/suppl/10.3171/2019. 12.FOCUS19448.

\section{Previous Presentations}

Portions of this work were presented in poster form at the International Neural Tube Defects Conference, Boston, Massachusetts, 2019.

\section{Correspondence}

Jonathan Castillo: Baylor College of Medicine, Houston, TX. jcporter@texaschildrens.org. 